ARCHIVO ESPAÑOL DE ARTE, LXXIX, 315

JULIO-SEPTIEMBRE, pp. 299-306, 2006

ISSN: 0004-0428

\title{
JUAN GUAS EN LA CATEDRAL DE SEGOVIA
}

\author{
POR \\ MARÍA LÓPEZ DÍEZ \\ Doctora en Historia del Arte
}

\begin{abstract}
Juan Guas es uno de los canteros más activos de la segunda mitad del siglo XV. Durante veinte años (14711491) trabajó en Segovia, donde participó en la construcción de la catedral y en otros edificios de la misma ciudad, siendo Segovia un lugar de referencia obligada en la investigación sobre el maestro. El presente artículo analiza, desde un punto de vista documental, la participación de Guas en las obras de la catedral a través de los diferentes contratos que firmó con el cabildo catedralicio. Estos permiten evaluar la progresiva consolidación de Guas en el ejercicio de su profesión, de gran interés para el mejor conocimiento de la práctica artística de su época.

Palabras clave: Juan Guas; arquitectura; Siglo XV; Segovia; catedral; Reyes Católicos.
\end{abstract}

Juan Guas, one of the most active stone masons of the second half of the 15th century, worked in Segovia for 20 years (1471-1491), where he participated in the construction of the Cathedral and other buildings in the city. Thus Segovia is a necessary place of reference for the study of this master. In this article the participation of Guas in the building of the Cathedral is analyzed from the documental point of view through the different contracts he signed with the Cathedral chapter. These documents allow for the evaluation of the progressive consolidation of Guas in the exercise of his profession, and are of great interest for the better understanding of artistic practice during his day.

Key words: Juan Guas; architecture; 15th century; Segovia; cathedral; Ferdinand and Isabel.

Juan Guas (14..-1496), maestro de cantería, es uno de los personajes clave para comprender el complejo entramado de la arquitectura castellana de finales del siglo XV. En los últimos tiempos, su figura ha sido objeto de una creciente atención debido al aumento de los estudios relacionados con esta época, en general, y con el reinado de los Reyes Católicos, en particular. Sin embargo, las líneas principales de investigación sobre Guas fueron establecidas por August Mayer y, sobre todo, por José María Azcárate, quien sigue siendo, hoy por hoy, el que ha realizado un mayor esfuerzo por aproximarse a la vida y obra del artista toledano ${ }^{1}$.

1 Aunque todavía no existe una monografía sobre el autor, su figura ha sido estudiada, entre otros, por Ceán Bermúdez, Juan Agustín, Diccionario Histórico de los más Ilustres profesores de las Bellas Artes en España, Madrid, 1800, 
La dificultad que entraña el estudio de un maestro de esta época estriba en el sistema de trabajo; un proceso que se centra básicamente en la ejecución de trazas y en la dirección de obra, de forma que, en el momento en que los dibujos no se conservan o que el edificio en cuestión haya sido alterado o desaparecido, es extremadamente complicado cifrar la intervención directa del artífice. Los problemas de autoría, además, se acrecientan si sumamos otros factores como son la uniformidad del lenguaje arquitectónico en este tiempo y la participación de diferentes canteros en distintos edificios a la vez. Por esta razón, muchas veces son las fuentes documentales, a pesar de sus condicionantes, las que ofrecen el método más práctico y certero para recomponer la autoría y trayectoria, tantas veces fragmentada, de estos maestros.

En 1947 el P. Arturo Hernández, archivero de la Catedral de Segovia, publicaba un artículo que ofrecía abundante información sobre el paso de Juan Guas por esta ciudad ${ }^{2}$. Partiendo de las anotaciones recogidas en los Libros de Fábrica, se ponía de manifiesto la importancia que Segovia tuvo en el ejercicio profesional del cantero. Sin embargo, una lectura más detenida de estos fondos catedralicios nos permite conocer con mayor exactitud los términos en los que se estableció la relación de Guas con la Catedral, mediante acuerdos sucesivos entre 1471 y $1491^{3}$.

No obstante, conviene recordar que el carácter de este tipo de documentos es estrictamente jurídico y que tienen, por tanto, obvias limitaciones en lo que a pormenores artísticos se refiere. En cambio, sí suponen una eficaz referencia a la hora de valorar la progresiva consolidación de la figura del maestro de obras y el consiguiente reparto de obligaciones entre artífice y cliente. Este aspecto queda ampliamente reflejado en el caso que nos ocupa, donde el análisis de los diferentes contratos de Guas, dos de los cuatro que firma con la catedral, constatan el cambio cualitativo que el maestro experimenta a lo largo de esos años.

La presencia de Juan Guas en la catedral de Segovia, entonces situada junto al Alcázar, se justifica en el contexto de las profundas iniciativas arquitectónicas emprendidas en la segunda mitad del cuatrocientos, coincidiendo, aproximadamente, con la prelatura del obispo don Juan Arias de Ávila (1461-1497). Este programa de reformas tiene como finalidad la transformación del templo románico en un edificio más acorde con las necesidades y criterios estéticos imperantes, planteando

II, p. 237; Conde de la Viñaza, Adicciones al diccionario de histórico de los más ilustres profesores de las Bellas Artes en España de don Juan Agustín Ceán Bermúdez, Madrid, 1889-1894, I, p. 79; Cotarelo, Emilio, "El licenciado Sebastián de Horozco y sus obras", en B.R.A.E., II, Madrid,1915, pp. 645-694; Ramírez de Arellano, Rafael, Catálogo de Artífices que trabajaron en Toledo y cuyos nombres y obras aparecen en los archivos de sus parroquias, Toledo, 1920; Mayer, Augusto L., "Guas", en Allgemeines Lexicon der bilbenden Künstler von der Antike bis zur gegenwarte, eds. Ulrich Thieme und Fred. C. Willis, Leipzig, 1922, XV, pp. 177-179; El Estilo Gótico en España, Madrid, 1960; Hernández, Arturo, "Juan Guas, maestro de obras de la catedral de Segovia. 1472-1491", en Boletín del Seminario de Arte y Arqueología, Valladolid, XLIII, 1947, pp. 57-100; Azcárate, José María, "Sobre el orígen de Juan Guas", en A.E.A., Madrid, XXIII, 1950, pp. 255-256; "La obra toledana de Juan Guas", en A.E.A., Madrid, XXIX, 1956, pp. 9-42; La Arquitectura Gótica Toledana del siglo XV, Madrid, 1958, p.71; "Sentido y significación de la Arquitectura hispano-flamenca en la corte de Isabel la Católica”, en B.S.A.A., Valladolid, XXXV, 1971, pp.201-223; Brans, J.V.L "Juan Guas, escultor", en Goya, Madrid, 36, 1960, pp.362-367; Gómez-Moreno, Manuel, Catálogo Monumental de la provincia de Ávila, Ávila, 1983; Marías Franco, Fernando, "Una carta de censo y tributo otorgada por Juan Guas", en B.S.E.A.A. Valladolid, XL-XLI, 1975, pp. 646-648; Domínguez Casas, Rafael, "San Juan de los Reyes: espacio funerario y aposento regio", en B.S.E.A.A., Valladolid, LVI, 1990, pp.364-383 y Arte y etiqueta de los Reyes Católicos. Artistas, residencias, jardines y bosques, Madrid, 1993, pp.42-44; Martínez Frías, José María, La huella de Juan Guas en la Catedral de Ávila, Ávila, 1998. Gómez Martínez, Javier, El Gótico Español en la Edad Moderna. Bóvedas de crucería, Valladolid, 1998; Abad Castro, Concepción, "Juan Guas y la capilla de 'La Piedad' en el convento de San Francisco de Ávila", en Anuario del Departamento de Historia y Teoría del Arte (U.A.M.), Madrid, XV, 2003, pp.29-44.

2 Hernández, Arturo, 1947

3 Archivo Catedral de Segovia. Registro de Pedro Fernández de Castro. 4-III-1476, ff. 131-131v; Libro de Fábrica, C-205, 7-VII-1480; publicados en López Diez, María, Los Trastámara en Segovia. Juan Guas, maestro de obras reales, Segovia, 2006, pp. 301-304 
actuaciones que afectan tanto a la estructura del edificio como a otros aspectos relacionados, hasta prácticamente rehacerlo por entero. Las obras continuarán entrado el siglo XVI. Sin embargo, en 1520, la catedral es cercada y minada durante el levantamiento comunero y, posteriormente, derrocada y trasladada al sitio que ahora ocupa, en la plaza mayor de la ciudad.

Así, en 1471, ante la decisión de construir un nuevo claustro, Guas es convocado por la catedral para responsabilizarse de su ejecución, mientras, en este mismo año, se ocupaba también en el claustro de la catedral de Ávila ${ }^{4}$. Las razones de su elección por parte del cabildo segoviano, más allá del acostumbrado intercambio de canteros entre catedrales, no están aún suficientemente aclaradas. En cualquier caso, Juan Guas se vinculará a las obras de la catedral de Segovia por espacio de veinte años (1471-1491), regulando su compromiso mediante una serie de contratos de cinco años de duración cada uno, por lo menos en lo que se refiere a los primeros. Solamente disponemos del segundo y tercer documento.

Son los acuerdos posteriores los que dan referencias del primer contrato (1471-1476). Por estos, podemos establecer que se hizo efectivo en seis de febrero de 1471, y no en 1472 como se consideraba hasta ahora. En cuanto a los términos del concierto, cabe pensar que serían muy parecidos a los reflejados en el contrato con la catedral de Ávila en 1471: 3.000 maravedís al año y veinte fanegas de trigo, $50 \mathrm{mrs}$ de jornal cada día de trabajo y casa para su aposento ${ }^{5}$. Pronto se debió hacer cargo de la obra, puesto que en el mes de abril se anota la preparación de la habitación de las trazas y en mayo consta que ya se está trabajando en el claustro ${ }^{6}$. Aunque la firma del contrato implicaba vivienda para el maestro, en un principio todo apunta a que se estableció a pie de obra, aprovechando algunas habitaciones del viejo palacio episcopal sobre el que se construiría el nuevo claustro y sus dependencias 7 . Habrá, pues, que esperar hasta 1475 para encontrar la primera referencia sobre la cesión de una casa en la canongía 8 .

En estos primeros cinco años de trabajo en Segovia, siguiendo la práctica común entre los canteros de este tiempo de emplearse en varias obras simultáneamente, Guas acomete la construcción del claustro segoviano, continúa con la actividad en Ávila y comienza en 1472 su colaboración en el monasterio jerónimo de Santa María del Parral, extramuros de la ciudad de

\footnotetext{
${ }^{4}$ Sobre su trabajo en la catedral abulense vide Gómez-Moreno, M., 1983, p. 83: Juan Guas se compromete en "las cosas que fueran nesçesarias de canteria, especialmente para que luego ponga obra de faser una capilla en la claustra"; Azcárate, J. Mª., 1958; Martínez Frías, J. Mª., 1998.

5 Gómez-Moreno, M., 1983, p. 89. Juan Guas trabajó por primera vez en la catedral de Ávila (1458-1463) en el traslado de la Puerta de los Apóstoles y, luego, como maestro en 1467, cobrando 1.000 mrs anuales hasta que en 1471 se fijan las condiciones citadas.

6 Archivo Catedral de Segovia. Libro de Fábrica, C-201. 19-IV-1471: "Costaron quinse tablas de chylla de a dose pies e çinco cabrios de a quinse pies e quarenta tablas orellanas para çerrar la sala de la traça e para un tejado dosientos e ochenta e çinco mrs"

20-V-1471: "Mandaron los señores dar de comer al obispo que desenbiolo la claustra quando se descalabraron los canteros costaron dos pares de gallinas çiento e veynte mrs"

${ }^{7}$ Para la construcción del claustro, Juan Arias de Ávila dona en 1472 los viejos palacios episcopales, junto a la cava del Alcázar, y levanta unos nuevos al otro lado de la catedral, más próximos a la canongía. Parte de la vieja construcción es absorbida para edificar el nuevo claustro que aquí nos ocupa, aprovechando algunas salas, como en las que se aposenta el maestro.

A.C.Sg. Libro de Fábrica, C-201. 20-II-1472: "Dy a dos maestros e a un peon que anduvo medyo dya a çerrar el postygo de las casas del maestro que se abryo para la yndulgençia que fiso el alcayde que se çerrase e labraron en la sala lxxxii"

8 A.C.Sg. Registro de Pedro de Castro. 27-XI-1475: "Mandaron que la casa que tenya Iñigo benefiçiado que fue que vaco por aber renunçiado su media raçion que es en la calongia que alquilaron casas en que mora Lope Martines e Alonso de .. que se de a Juan Guas cantero maestro de la obra de la fabrica e de la claustra en que more en tanto que la voluntad del cabillo fuere e por el presçio que la tenya el dicho Yñigo e quel presçio e pension pague la obra de la fabrica e las pitanças Testigos Juan Marcos e Diego del Castillo e Pedro de Fierro medios raçioneros mandaron a Pero Alonso que la faga e libre", f. 122v
}

AEA, LXXIX, 315, JULIO-SEPTIEMBRE, 299-306, 2006, ISSN: 0004-0428 
Segovia, que se prolongará prácticamente hasta el final de sus días9. Desde 1475 entra al servicio de la familia de los Mendoza, participando en el castillo del Real de Manzanares y en el Palacio del Infantado de Guadalajara10.

El segundo contrato (1476-1481) nos lo ofrecen los Registros Capitulares o Libro de Acuerdos del Cabildo, que guardan una copia fidedigna del contrato original. Fechado en 4 de marzo de 1476, Juan Guas se obliga desde el 6 de febrero de dicho año hasta un lustro después para "...servyr e estar por maestro en el edifiçio e obra que se fase en la claustra de la dicha yglesia cathedral...". Este acuerdo verifica la vecindad de Guas en Segovia, lo que supone un dato de suma importancia, ya que hasta ahora solo constaba la de Toledo, y que fije su residencia en Segovia no deja de ser una elocuente prueba de su sólido asentamiento en esta ciudad. En lo que se refiere a su posición, se precisa que no se le toma como maestro de obras de toda la Fábrica, sino sólo de la claustra. No se especifican las características de su trabajo, como ocurre en otros contratos contemporáneos, aunque si las condiciones.

La catedral se compromete a pagarle un sueldo anual de tres mil maravedís de la moneda en curso, el pan del maestro que consistía en veinte fanegas de buen trigo al año, ambas retribuciones repartidas en tres tercios a contar desde la fecha del contrato, casa y, por cada jornada de trabajo, cincuenta mrs. Por su parte, el maestro, responsable también de los jornales de los oficiales, se obliga a tener siempre tres mozos en la obra, quienes cobrarán según su capacidad a vista de ofiçiales del dicho ofiçio. Debe dirigir el trabajo dando órdenes a los oficiales para que se hagan cargo de la obra y sólo podrá ausentarse de la catedral por tiempo máximo de un mes, siempre con la licencia del deán y cabildo. En el caso de que partiera, se compromete a dejar a un oficial al frente de la obra con las instrucciones pertinentes. Si el claustro se acabara antes del vencimiento del contrato, Juan Guas recibiría cinco mil mrs. de merced. En cambio, si la catedral decidiera suspender las obras, el maestro seguiría cobrando sus tercios correspondientes hasta la expiración del acuerdo. En este último supuesto, cuando la catedral decidiera reanudar la actividad, el maestro se obligaba a acudir antes de un mes. El testigo por parte del contratado es el entallador Martín de Lizcano ${ }^{11}$.

De esta escritura se deduce que el cometido del maestro de obras es organizar el trabajo tanto desde un punto de vista proyectual, mediante la realización de las trazas, como desde un aspecto práctico que engloba la sistematización del trabajo, el cálculo y control de costes o la labor propia de cantero. Por esta razón, la escritura hace hincapié en que recibiría 50 mrs de jornal por cada "dia de los que fueren de labrar e el andovyere en la dicha obra por su persona".

En lo que concierne al tercer acuerdo, presenta sensibles diferencias con respecto al anterior, sobre todo, en lo que a la valoración profesional de Guas se refiere. En primer lugar, Juan Guas por estos años había consolidado plenamente su carrera y era ya uno de los canteros más demandados de su tiempo. El maestro había conseguido ampliar considerablemente no solo el área geográfica de sus encargos, sino también el tipo de clientela, que cubría los principales estamentos: Iglesia, corona y nobleza. Así, en torno a los 80, aparte de su trabajo en diferentes edificios de Segovia, había obtenido el título de maestro mayor de las obras del rey e reyna, como se hace

\footnotetext{
${ }^{9}$ Bosarte, Isidro., Viaje artístico a varios pueblos de España, (1804), I, Madrid, 1978, pp.353-354.

10 Azcárate, J.Mª., 1958

${ }^{11}$ Martín de Lizcano, entallador, trabajó en la catedral de Segovia desde 1483 a 1486. Según noticias extraídas de la misma catedral, trabajó posteriormente en la Cartuja de Miraflores, Burgos, y en la de El Paular, Rascafría.

A.C.Sg. Libro de Fábrica, C-204. 10-16-X-1486: "Pague por dos tablas juntadas y encoladas con sus travyesas de olmo aserradas a Martyn de Lizcano quando se partya para Burgos a Miraflores çinquenta mrs l”, f. 113v

Libro de Fábrica, C-206. Semana 18-V-1489: "A Pedro criado de Martyn entallador que le ayudo de çinco dyas que labro que el sabado fue a Rascafria a Martyn que estaba ende de los dichos çinco ccl dyas"
} 
constar en el documento; el proyecto de San Juan de los Reyes en Toledo (1477) acababa de comenzar, también el de la hospedería de Guadalupe, mantiene sus trabajos en Ávila, continúa a las órdenes de los Mendoza e, incluso, parece posible su participación en la iglesia de La Mejorada, Olmedo (1476) ${ }^{12}$.

No obstante, este documento presenta una circunstancia que acrecienta su interés. La cuestión es que el segundo contrato vencía en febrero de 1481, cumpliendo el plazo quinquenal, y este tercero se fecha en julio de 1480. La razón del anticipo viene dada porque el claustro para el que se había contratado a Guas, por lo menos en lo principal, estaba concluido en 1479, aproximadamente más de un año antes de la fecha prevista, lo que suponía una prima de cinco mil mrs para el maestro. Por lo tanto, ajustándonos a la letra, el contrato está cumplido y la iglesia "no tiene derecho ny facultad para que pueda labrar".

No obstante, lo que realmente llama la atención es que este no es un contrato por obra, sino un acuerdo de vinculación de Guas a la catedral, sin que exista ninguna obligación de trabajar en el templo, sino de estar a disposición del mismo por si es nuevamente requerido. Es decir, que la mesa capitular le ofrece una serie de remuneraciones anuales con la única finalidad de que el cantero no se desligue completamente de la iglesia. Para ello, apelan a la "afeçion que tiene a esta yglesia e grandes serviçios que le ha fecho", se le reconoce el buen trabajo que ha realizado y están dispuestos a pagarle anualmente dos mil mrs de quitación y doce fanegas de trigo y respetarle la casa de la Canongía mientras permanezca en la ciudad. La intencionalidad que subyace en este documento pone de relieve la nueva situación del artífice en relación con el contrato anterior: Juan Guas ha logrado situarse en una posición ciertamente ventajosa.

En lo tocante a las obligaciones del cantero, estas se refieren básicamente a su disponibilidad. Guas debe acudir a la llamada del cabildo, ya sea para labrar o para dar consejo, en un plazo de treinta días y si se decide la necesidad de su trabajo, se respetarán las condiciones y salarios de los contratos anteriores. Una de las mejoras con respecto a los acuerdos precedentes es que ahora le pagan a precio de jornal, $50 \mathrm{mrs}$., cada día de desplazamiento entre la catedral de Segovia y el lugar donde estuviera trabajando en ese momento, a menos que él dejara la ciudad por su propia voluntad. La casa que habitaba en la Canongía Nueva se le respeta pero sólo treinta días antes de su llegada. Nada más partir, el cabildo podría disponer de ella. Los testigos por parte de Guas son los canteros Juan de la Hoz y Pedro Polido, quien con Guas y Bonifacio trabajó en El Parral y, esporádicamente, también en la catedral de Segovia ${ }^{13}$. Al final del documento se incluye una nota posterior en que queda registrado el recibo de los cinco mil mrs. de bonificación por la conclusión del claustro, en tres de julio de 1481, un año más tarde de la firma del presente acuerdo ${ }^{14}$.

\footnotetext{
12 Azcárate, J. Mª , 1958. Sobre la posible participación de Guas en La Mejorada, cfr. Ruiz Hernando, Antonio, Los Monasterios Jerónimos Españoles, Segovia, 1997, p. 498

13 Polido, aunque segoviano, se formó en el taller de Hanequín de Bruselas en la catedral de Toledo, constando su participación como oficial en la ejecución de la Puerta de los Leones en 1452 y 1453 . Tras su labor en la catedral primada vuelve a Segovia donde aparece en la Catedral y trabajará con Juan Guas y Bonifacio en la construcción de El Parral (1472-1484) y Santa Cruz. Vuelve a trabajar en la catedral de Segovia en 1480 y este mismo año firma como testigo en el tercer contrato de Guas con la catedral. El 7 de mayo de 1491, cede el censo de unas casas en Segovia al también cantero Francisco Sánchez de Toledo y ya en 1497 participará con Juan de la Riba en las obras del colegio de Santa Cruz en Valladolid, obra financiada por el Cardenal Cisneros. Cfr. Azcárate, J. Mª., 1958; "Análisis estilístico de las formas arquitectónicas de la Puerta de los Leones de la Catedral de Toledo", en Homenaje al profesor Cayetano de Mergelina, Valencia, 1962, pp. 97-122; Gómez-Moreno, M., "Sobre el Renacimiento en Castilla I", en A.E.A.A., Madrid, I, 1925, p. 7; . Sobre el censo de sus casas, Ruiz, J.A., Historia del urbanismo en la ciudad de Segovia, Madrid, 1982, II, p.9.

14 Esta prima también consta en la consiguiente anotación de los Libros de Fábrica pero con fecha del 24 del mismo mes. A.C.Sg. Libro de Fábrica, C-205. 24-VIII-1481: "En xxiiii de jullio del dicho año di a Juan Guas çinco myll mrs que se le devyan de la merçed de los señores dean e cabildo le fisieron de los dos contratos segund esta asentado en una hoja en fin de este libro e di su pago ansy mesmo ante Pero Ferrandes de Castro montaron vM"
} 
En el año de 1480 recibe los mrs y el pan de maestro convenido en los contratos anteriores, $3.000 \mathrm{mrs}$ anuales y veinte fanegas de trigo, por lo que se puede establecer que el acuerdo de julio de 1480 tiene validez a partir del año siguiente, en enero del 815. La situación cambia desde este año. No se han encontrado más contratos o acuerdos explícitos, pero las anotaciones de la Fábrica siguen arrojando impagables datos para evaluar la situación. De lo que ocurre durante 1481 y 1482, años de muy poca actividad constructiva en la catedral segoviana, sabemos por una referencia de 1484 que alude a lo que se adeudaba a Guas. Aquí se le cita como maestro de la obra, aunque las cantidades que se registran son un tanto equívocas ${ }^{16}$. No así en lo que afecta a 1483 , fecha en la que se reactivan sensiblemente los trabajos y donde queda registrado con mayor claridad que percibe dos mil mrs anuales y catorce fanegas de trigo ${ }^{17}$. Estas cifras se corresponden con el acuerdo al que se llegó en 1480 y que se respetó en lo fundamental, tanto en el jornal diario como en las quitaciones anuales, con la salvedad de que el pan del maestro son dos fanegas más de las pactadas en principio.

De nuevo, en 1484 el maestro, que este mismo año se reincorpora a la catedral de Toledo en calidad de aparejador, avanza un paso más en su relación con el templo segoviano. Seguramente establecido mediante contrato, el cabildo acuerda pagarle $4.000 \mathrm{mrs}$ anuales, más veinte fanegas de trigo, aparte de los desplazamientos, casa, que el cantero exige que esté dispuesta para sus estancias en Segovia, y los consabidos $50 \mathrm{mrs}$. de jornal ${ }^{18}$. Esta subida de $1.000 \mathrm{mrs}$ se mantendrá hasta el fin de su trabajo en la catedral, recibiendo, no siempre con puntualidad, sus quitaciones anuales. Hay que señalar que los tercios correspondientes a 1488 se le pagan mediante el cantero Francisco de Ávila, aparejador del segoviano monasterio de Santa Cruz, y el jerónimo fray Gil,

15 A.C.Sg. Libro de Fábrica, C-205. 7-VII-1480: "En syete dyas del mes de jullio del dicho año dio el dicho secretario a Juan Guas por mandado de los señores dean e cabildo dos myll e dos mrs para en cuenta e pago de los tres myll mrs que se le devyan de su quitaçion deste año de ochenta que se cunplira en fin del mes de enero primero que verna hanse le dar mas veynte fanegas de trigo las quales mando Juan Guas dar a Pero Alfonso"

11-XI-1480: "En xi dias del mes de octubre del dicho año de lxxx di a Juan Guas nueveçientos e noventa e ocho mrs con que se le acabaron de pagar los tres myll mrs que se le devyan de su quitaçion del dicho año de lxxx"

16 A.C.Sg. Libro de Fábrica, C-204. 5a Semana-III-1"-IV-1484: "Pague a Juan Guas maestro de la obra del alcançe que fiso a los señores arçediano de Cuellar y Anton Vasques y secretario y a mi de años pasados de lxxxi y lxxxii de su quitaçion y de veynte fanegas de trigo que le devyan de ante feneçiose cuenta que fiso de alcançe a los susodichos syete myll e quatroçientos e setenta e dos mrs pagueselos en xx doblas y lo otro en reales viiMcccclxxii”, f.77

17 A.C.Sg. Libro de Fábrica, C-206. 8-IX-1483: "Pague al dicho Juan Guas de dos terçios de su quitaçion fasta fin de agosto de dos myll mrs que montan en ellos iMcccxxxiii iiR

Paguelo mas de los dichos dos terçios quatorse fanegas de trigo de su quitaçion que costaron a lxxv iMl"

30-XII: "Pague a Juan Guas maestro de las obras desta iglesia de un terçio de dos myll mrs que tiene de su quitaçion deste terçio que se acabo en fin de disyenbre seysçientos y sesenta y seys mrs e iiii reales dclxvi iiii

Paguele mas seys fanegas de trigo del dicho terçio postrimero que costaron a (en blanco)"

18 A.C.Sg. Libro de Fábrica, C-204. $3^{\text {a }}$ Semana-VIII-1484: "Paguele al dicho Juan Guas del terçio de fin de abril y deste de fin de agosto de su quitaçion de quatro myll mrs que se le han de dar en cada un año de prinçipio deste dicho año adelante acordado por los señores arçediano de Cuellar y Anton Vasques y el secretario y yo con el dicho Juan Guas y veynte fanegas de trigo paguele de los dichos dos terçios dos myll y seisçientos y sesenta y seys mrs y quatro cornados y todos sus jornales y todas cuentas de entre el y mi fasta en fin del dicho mes de agosto pagadas salvo que le quedo devyendo seys fanegas de trigo del terçio de Nabydad pasado y quatorse fanegas de los terçios de fin de abril y de fin de agosto que son veynte fanegas de trigo levo el remate desta dicha cuenta en su memorial para asentar en su libro iimdclxvi iiii", f. 79v

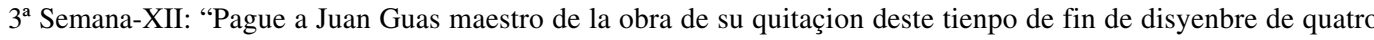
myll mrs que le dan cada año myll e tresyentos e treynta e tres mrs e dos reales imcccxxxiiii ii $r$

Paguele mas por veynte fanegas de trigo que se le devyan del convynese a quatro reales la fanega que montan en ellas ochenta reales que se convenyeron en el año de lxxxv en abril que non se le habya pagado iimcccclxxx

Pague mas por el alquiley de la casa de Juan Guas que saco Anton Vasques para el por myll e çien mrs que monta en ocho meses e medio que la tuvo el dicho Juan Guas disyendo que se queria aposentar en ella e tener alli una cama $\mathrm{y}$ una ama para quando vinyese fasta que la tomo Monjaras en fin de junyo dcclxxix e medio", f. 82v 
profeso en Santa María de El Parral, mediador en muchas obras del maestro y que también se hará cargo del finiquito de Guas ${ }^{19}$. Esto tiene lugar en 1491, cuando la catedral prescinda definitivamente de los servicios del maestro ante la ausencia de iniciativas arquitectónicas ${ }^{20}$.

De todos estos años de trabajo profesional únicamente conservamos el claustro gracias a que fue trasladado en 1524 a la nueva catedral que diseñaron los Hontañón. El resto de sus intervenciones sólo subsisten por la documentación que hace constar la importante labor llevada a cabo por el maestro.

Es evidente el progreso profesional que experimenta el maestro desde el primer contrato de 1471 hasta 1491, año de su despido. En estos veinte años y a través de los sucesivos acuerdos, se observa que la trayectoria de Juan Guas comienza con unas condiciones fijadas por la catedral, respetando los términos habituales en esta época, y que se ven rápidamente superadas por la eficacia del maestro. Así, en los documentos posteriores, aparte de mejorar las contraprestaciones económicas, existe una mayor fuerza por parte de Guas a la hora de fijar su postura frente al cliente. Prueba de ello es el acuerdo de vinculación de 1480. Además, estos logros no sólo se ciñen al ámbito profesional, puesto que van acompañados de un afianzamiento de su posición social y que

19 A.C.Sg. Libro de Fábrica, C-204. 19-24-XII-1485: "Pague a Juan Guas maestro de la obra de dos terçios de su quitaçion de los quatro myll mrs que le dan convyene a saber del terçio de fin de agosto e del fin de disyenbre deste dicho año de lxxxv años dos myll y seys çientos y sesenta e seys mrs e quatro cornados de los dichos dos terçios

Paguele mas veynte fanegas de trigo deste dicho año que se convynieron çiento e veynte quatro mrs la fanega que non se pudo convenyr menos o que le dyesen su trigo y valya a çiento y quarenta y ocho mrs que montan ochenta reales que valen dos myll e quatroçientos e ochenta mrs", f. 97

16-VI-1486: "En dies y seys de junyo fenesçimos cuenta Juan Guas y yo de todas cosas fasta oy dicho dya ansy del trigo de su quitaçion siete fanegas a cc de los dyneros de la dicha su quitaçion y de todos los jornales y cosas fasta oy dicho dya pagadas y con syete fanegas de trigo deste terçio de fin de abril proximo pasado y myll y tresyentos y treynta y tres mrs y dos reales de su quitaçion del dicho terçio que me cargo quedamos libres y quitos fasta oy dicho dya levo en memorial para poner en su libro otro tanto imcccxxx", f. 110

27-XI-3-XII-1486: "Pague a Juan Guas maestro de la obra de su quitaçion del terçio de fin de agosto myll y tresyentos y reynta y tres mrs y dos cornados imcccxxxiii y ii cornados

Paguele mas por syete fanegas de trigo del dicho terçio de fin de agosto que valyan a çien mrs que montan dcc", f.115

18-24-XII: "Pague a Juan Guas maestro de la obra del terçio de su quitaçion de fin de disyenbre de los quatro myll mrs que se le dan en cada año myll y tresyentos y trynta y tres mrs y dos reales ivmdccccxl

Paguele mas por seys fanegas de trigo que se le devyan del dicho terçio de las veynte fanegas de trigo que se le dan cada un año sobre catorse que tenya resçibydas del año a çien mrs la fanega que montan seisçientos mrs", f. 115v

Libro de Fábrica, C-206. 23-IV-1487. Margen: "Vyno Juan Guas paguele del terçio de fyn de disyenbre iMcccxxxiii e ii cornados del de iv de abril iMcccxxxxiiii e ii cornados quatorse fanegas de trigo a cxxiiii de sus jornales y venydas veynte e dos dyas a 1 deste camyno tres dyas de venyda e çinco desta va para los moldes y ver y dar orden"

4-VI-1487: "Pague a Juan Guas de su quitaçion del terçio de Nabydad que non la dy en cuenta el año pasado myll y tresyentos y treynta y tres mrs y dos cornados

Paguele mas por seys fanegas de trigo del dicho terçio de su quitaçion a quatro reales la fanega que son (en blanco)

Pague mas al dicho Juan Guas del terçio de fin de abril deste dicho año de lxxxvii de su quitaçion myll y tresyentos y treynta e tres mrs e dos cornados iMcccxxxiii iicrs

Paguele mas por syete fanegas de trigo del dicho terçio a quatro reales la fanega que montan dccclxvii

De los borseguyes clxv"

5-V-1488: "Pague a Juan Guas maestro de la obra del terçio que se acabo en fin de abril de su quitaçion de los quatro myll mrs que le dan en cada un año myll e tresyentos e treynta e tres mrs y dos cornados mas syete fanegas de trigo a quatro reales iMcccxxxiii iicrs

Dyle agora que me dyo Dyego de Madrigal para este terçio y el pasado iMd

Dyle a Françisco de Avyla por su mandado iiiicrs

Pague a Fray Gil por su mandado de los dos terçios del año de lxxx y viii de su quitaçion convyene a saber de fin de agosto y de fin de disyenbre de todo el año de lxxxix viMdevi e iiii crs e mas xl fanegas de trigo de los dichos dos con que soy libre de el"

20-X-1488: "A Juan Guas maestro de la obra del terçio que se acabo en fin de agosto que agora paso de los quatro myll mrs que le dan en cada un año iMcccxxxiiii

AEA, LXXIX, 315, JULIO-SEPTIEMBRE, 299-306, 2006, ISSN: 0004-0428 
tienen como colofón que el maestro comprara una capilla en la toledana iglesia de San Justo y San Pastor para su enterramiento, algo inusual en un maestro de cantería de finales del XV.

Parece, a la vista de lo expuesto, que Juan Guas, si nunca perdió la constante referencia de Toledo, su ciudad de origen y destino, encontraría en Segovia la plataforma adecuada para desarrollar su ascendente carrera de cantero. No sólo porque en esta ciudad, que acoge el mayor volumen de obra suya, es en la que pasó más años trabajando, sino porque es aquí donde forma y reúne un eficaz taller en torno a las obras catedralicias. Taller que acompañará al cantero en sus otros encargos y que, a la muerte de Guas, asumirá la construcción de los proyectos más interesantes de Castilla, difundiendo el estilo y enseñanzas aprendidas del maestro.

Recibido: 22-VI-2004

Aceptado: 27-IX-2004

Paguele mas por en blanco fanegas de trigo del dicho terçio de fin de agosto (en blanco) mrs la fanega que montan (en blanco)"

29-XII-1488: "Pague a Juan Guas maestro de la obra del terçio que se cumple en fin de disyenbre deste año de lxxxviii de su quitacion de los quatro myll mrs que le dan en cada un año iMcccxxxiii iicrs

Paguele mas por seys fanegas de trigo del dicho terçio de las veynte fanegas que le dan cada año a çien mrs que montan seisçientos mrs"

25-V-1489: "A Juan Guas del terçio de fin de abril iMcccxxxiii iicrs

Mas de syete fanegas de trigo"

21-XII-1489: "Pague a Juan Guas maestro de la obra del terçio de fin de dysienbre de su quitaçion de los quatro myll mrs que le dan cada un año myll y tresientos treynta y tres mrs y dos cornados

Paguele mas por siete fanegas de trigo del dicho terçio e tres reales que montan seisçientos y quarenta y un mrs"

20 A.C.Sg. Libro de Fábrica, C-208, 24-II-1491: " Mas di esto susodicho a un correo que llevo cartas al señor liçençiado Quintanapalla e a Juan Guas para despedir al dicho Juan Guas nueve reales son cclxxix"

Hernández, A. , 1947, p. 67: "Mas di a fray gil del parral de segovia por juan guas quatro mill marauedis que la fabrica dela iglesia de segovia le avia de dar de su salario hasta el año de XCI, que lo mandaron espedir los señores dela dicha iglesia, porque non le avian menester, porque no se labraba en la dicha iglesia, degelo en fin de diciembre de $\mathrm{XCI}^{\circ}$ " 